九州大学学術情報リポジトリ

Kyushu University Institutional Repository

\title{
A numerical verification method of bifurcating solutions for 3-dimensional Rayleigh-B' enard problems
}

Kim, Myoungnyoun

Faculty of Mathematics, Kyushu University

Nakao, Mitsuhiro T.

Faculty of Mathematics, Kyushu University

Watanabe, Yoshitaka

Computing and Communications Center, Kyushu University

Nishida, Takaaki

Faculty of Science and Engineering, Waseda University

http://hdl. handle. net/2324/4024

出版情報 : 2007-02-20. 九州大学大学院数理学研究院 バージョン：

権利関係 : 


\title{
MHF Preprint Series
}

Kyushu University

21st Century COE Program

Development of Dynamic Mathematics with

High Functionality

\section{A numerical verification method of bifurcating solutions for 3-dimensional Rayleigh-Bénard problems}

\author{
M. Kim, M. T. Nakao \\ Y. Watanabe, T. Nishida
}

MHF 2007-10

( Received February 20, 2007 )

Faculty of Mathematics

Kyushu University

Fukuoka, JAPAN 


\title{
Myoungnyoun Kim · Mitsuhiro T. Nakao · Yoshitaka
}

Watanabe · Takaaki Nishida

\section{A numerical verification method of bifurcating solutions for 3-dimensional Rayleigh-Bénard problems}

\begin{abstract}
This paper is the three dimensional extension of the two dimensional work [4] and [7] on a computer assisted proof of the existence of nontrivial steady state solutions for Rayleigh-Bénard convection based on the fixed point theorem using a Newton like operator. The differences are emerging of complicated types of bifurcation, direct attack on the problem without stream functions, and increased complexity of numerical computation. The last one makes it hard to proceed the verification of solutions corresponding to the points on bifurcation diagram for three dimensional case. Actually, this work should be the first result for the three dimensional Navier-Stokes problems which seems to be very difficult to solve by theoretical approaches.
\end{abstract}

\section{Introduction}

The Rayleigh-Bénard convection describes the instability of fluid between two infinite solid plates with hot bottom and cool top. The motion of fluid is self-sustained as soon as gravitational energy release overcomes dissipation losses, which is called Rayleigh's mechanism by buoyancy (for detailed mechanism, see [1] and [3]). We will use the Oberbeck-Boussinesq equations as approximate equations for this convection problem after normalization of variables and parameters:

$$
\begin{aligned}
\frac{1}{\mathscr{P}}\left[\frac{\partial \mathbf{u}}{\partial t}+(\mathbf{u} \cdot \nabla) \mathbf{u}\right]+\nabla p & =\Delta \mathbf{u}-(\mathscr{G}-\mathscr{R} T) \mathbf{e}_{z}, \\
\nabla \cdot \mathbf{u} & =0, \\
\frac{\partial T}{\partial t}+(\mathbf{u} \cdot \nabla) T & =\Delta T,
\end{aligned}
$$

where $\mathbf{u}=(u, v, w)$ is the velocity field, $p$ the pressure, $T$ the temperature, $\mathscr{R}$ Rayleigh number, $\mathscr{P}$ Prandtl number, and a parameter $\mathscr{G}$ containing gravity factors. We use stress free boundary conditions on the velocity field and Dirichlet boundary conditions on the temperature $\left(T_{z=0}=0, T_{z=\pi}=\pi\right)$. Under a reference pressure $p_{a}$, the equilibrium state comes from the pure heat conduction:

$$
\mathbf{u}=\mathbf{0}, \quad T=\pi-z, \quad p=\mathscr{G}(\pi-z)-\frac{1}{2} \mathscr{R}(\pi-z)^{2}+p_{a} .
$$

Myoungnyoun Kim

Faculty of Mathematics, Kyushu University 33, Fukuoka 812-8581, Japan

E-mail: mnkim@math.kyushu-u.ac.jp

Mitsuhiro T. Nakao

Faculty of Mathematics, Kyushu University 33, Fukuoka 812-8581, Japan

E-mail: mtnakao@math.kyushu-u.ac.jp

Yoshitaka Watanabe

Computing and Communications Center, Kyushu University 33, Fukuoka 812-8581, Japan

E-mail: watanabe@cc.kyushu-u.ac.jp

Takaaki Nishida

Faculty of Science and Engineering, Waseda University, 3-4-1 Okubo, Shinjuku-ku, Tokyo 169-8555, Japan

E-mail: tkknish@waseda.jp 
Using the conduction solution (2) and eliminating time derivatives from (1), we obtain the steady state bifurcation equations for the perturbation $(\mathbf{u}, \theta, p)$ to the equilibrium:

$$
\begin{aligned}
-\Delta \mathbf{u}+\frac{1}{\mathscr{P}}(\mathbf{u} \cdot \nabla) \mathbf{u}+\nabla p-\mathscr{R} \theta \mathbf{e}_{z} & =\mathbf{0}, \\
\nabla \cdot \mathbf{u} & =0 \\
-\Delta \theta+(\mathbf{u} \cdot \nabla) \theta-w & =0 .
\end{aligned}
$$

Given positive wave numbers $a, b \leq 1$, we assume that all fluid motions are essentially confined to

$$
\Omega=\left\{(x, y, z) \in \mathbb{R}^{3}: 0 \leq x \leq \frac{2 \pi}{a}, 0 \leq y \leq \frac{2 \pi}{b}, 0 \leq z \leq \pi\right\}, \quad|\Omega|=\frac{4 \pi^{3}}{a b},
$$

and impose parity conditions on new boundaries as in [5] together with periodic boundary conditions in horizontal directions [4]. From these boundary conditions, the velocity field, the perturbations of temperature and pressure can be represented by the Fourier series [5]:

$$
\mathbf{u}=\sum_{\alpha \neq \mathbf{0}}\left[u_{\alpha} \phi_{1}^{\alpha}, v_{\alpha} \phi_{2}^{\alpha}, w_{\alpha} \phi_{3}^{\alpha}\right], \quad \theta=\sum_{\alpha_{3} \neq 0} \theta_{\alpha} \phi_{3}^{\alpha}, \quad p=\sum_{\alpha \neq \mathbf{0}} p_{\alpha} \phi_{4}^{\alpha}
$$

where $\alpha \equiv\left(\alpha_{1}, \alpha_{2}, \alpha_{3}\right)$ is the three dimensional multi-index of non-negative integers $\mathbb{Z}_{0}$, and $u_{\alpha}, v_{\alpha}, w_{\alpha}, \theta_{\alpha}$ $p_{\alpha}$ are coefficients of $\mathbf{u}, \theta, p$ with respect to the base functions $\phi_{i}^{\alpha}$ defined by,

$$
\begin{aligned}
& \phi_{1}^{\alpha}(x, y, z)=K_{\alpha} \sin \left(a \alpha_{1} x\right) \cos \left(b \alpha_{2} y\right) \cos \left(\alpha_{3} z\right), \phi_{2}^{\alpha}(x, y, z)=K_{\alpha} \cos \left(a \alpha_{1} x\right) \sin \left(b \alpha_{2} y\right) \cos \left(\alpha_{3} z\right), \\
& \phi_{3}^{\alpha}(x, y, z)=K_{\alpha} \cos \left(a \alpha_{1} x\right) \cos \left(b \alpha_{2} y\right) \sin \left(\alpha_{3} z\right), \phi_{4}^{\alpha}(x, y, z)=K_{\alpha} \cos \left(a \alpha_{1} x\right) \cos \left(b \alpha_{2} y\right) \cos \left(\alpha_{3} z\right),
\end{aligned}
$$

where the normalization factor with respect to the usual $L^{2}(\Omega)$ inner product $\langle\cdot, \cdot\rangle$ is

$$
K_{\alpha}=\sqrt{\left(2-\delta_{0 \alpha_{1}}\right)\left(2-\delta_{0 \alpha_{2}}\right)\left(2-\delta_{0 \alpha_{3}}\right) /|\Omega|}, \quad \delta_{i j}=\text { Kronecker delta on } i, j .
$$

The various kinds of norms for $\mathbf{u}, \theta$ and $p$ in (4) can be written as:

$$
\begin{aligned}
& \|\mathbf{u}\|_{0}^{2}=\sum_{\alpha \neq \mathbf{0}}\left\{u_{\alpha}^{2}+v_{\alpha}^{2}+w_{\alpha}^{2}\right\},\|\nabla \mathbf{u}\|_{0}^{2}=\sum_{\alpha \neq \mathbf{0}}\left\{u_{\alpha}^{2}+v_{\alpha}^{2}+w_{\alpha}^{2}\right\} A_{\alpha}^{2},\left\|\nabla^{2} \mathbf{u}\right\|_{0}^{2}=\sum_{\alpha \neq \mathbf{0}}\left\{u_{\alpha}^{2}+v_{\alpha}^{2}+w_{\alpha}^{2}\right\} A_{\alpha}^{4}, \\
& \|\theta\|_{0}^{2}=\sum_{\alpha_{3} \neq 0} \theta_{\alpha}^{2}, \quad \quad\|\nabla \theta\|_{0}^{2}=\sum_{\alpha_{3} \neq 0} \theta_{\alpha}^{2} A_{\alpha}^{2}, \quad\left\|\nabla^{2} \theta\right\|_{0}^{2}=\sum_{\alpha_{3} \neq 0} \theta_{\alpha}^{2} A_{\alpha}^{4}, \\
& \|p\|_{0}^{2}=\sum_{\alpha \neq \mathbf{0}} p_{\alpha}^{2}, \quad \quad\|\nabla p\|_{0}^{2}=\sum_{\alpha \neq \mathbf{0}} p_{\alpha}^{2} A_{\alpha}^{2}, \quad\left\|\nabla^{2} p\right\|_{0}^{2}=\sum_{\alpha \neq \mathbf{0}} p_{\alpha}^{2} A_{\alpha}^{4},
\end{aligned}
$$

where $A_{\alpha} \equiv \sqrt{\left(a \alpha_{1}\right)^{2}+\left(b \alpha_{2}\right)^{2}+\alpha_{3}^{2}}$ provided that the corresponding righthand sides converge.

We now define the divergence free and orthogonal system by:

$$
\Phi^{\alpha}=\left[-\frac{a \alpha_{1} \alpha_{3}}{A_{\alpha} B_{\alpha}} \phi_{1}^{\alpha},-\frac{b \alpha_{2} \alpha_{3}}{A_{\alpha} B_{\alpha}} \phi_{2}^{\alpha}, \frac{B_{\alpha}}{A_{\alpha}} \phi_{3}^{\alpha}\right], \alpha \in I_{1}, \quad \Psi^{\alpha}=\left[\frac{b \alpha_{2}}{B_{\alpha}} \phi_{1}^{\alpha},-\frac{a \alpha_{1}}{B_{\alpha}} \phi_{2}^{\alpha}, 0\right], \alpha \in I_{2},
$$

where $B_{\alpha} \equiv \sqrt{\left(a \alpha_{1}\right)^{2}+\left(b \alpha_{2}\right)^{2}}$ and indices subsets are $I_{1} \equiv\left\{[1,0,1]+\mathbb{Z}_{0}^{3}\right\} \cup\left\{[0,1,1]+\mathbb{Z}_{0}^{3}\right\}, I_{2} \equiv[1,1,0]+$ $\mathbb{Z}_{0}^{3}$. Set $I_{0}=I_{1} \cup I_{2}$ and then define the function spaces $V$ and $W$ with associated usual $H^{1}-$ norm as follows:

$$
\begin{aligned}
& V=\left\{\mathbf{u}=\sum_{\alpha \in I_{0}}\left\{\xi_{\alpha} \Phi^{\alpha}+\eta_{\alpha} \Psi^{\alpha}\right\}:\|\Delta \mathbf{u}\|_{0}<\infty\right\} \subset H^{2}(\Omega)^{3}, \\
& W=\left\{\theta=\sum_{\alpha \in I_{3}} \theta_{\alpha} \phi_{3}^{\alpha}:\|\Delta \theta\|_{0}<\infty\right\} \subset H^{2}(\Omega), \quad \text { where } I_{3} \equiv[0,0,1]+\mathbb{Z}_{0}^{3} .
\end{aligned}
$$

Note that $\|\mathbf{u}\|_{0}^{2}=\sum_{\alpha \in I_{0}}\left\{\xi_{\alpha}^{2}+\eta_{\alpha}^{2}\right\},\|\nabla \mathbf{u}\|_{0}^{2}=\sum_{\alpha \in I_{0}}\left\{\xi_{\alpha}^{2}+\eta_{\alpha}^{2}\right\} A_{\alpha}^{2},\|\Delta \mathbf{u}\|_{0}^{2}=\sum_{\alpha \in I_{0}}\left\{\xi_{\alpha}^{2}+\eta_{\alpha}^{2}\right\} A_{\alpha}^{4}$ for all $\mathbf{u} \in V$, and $\|\theta\|_{0}^{2}=\sum_{\alpha \in I_{3}} \theta_{\alpha}^{2},\|\nabla \theta\|_{0}^{2}=\sum_{\alpha \in I_{3}} \theta_{\alpha}^{2} A_{\alpha}^{2},\|\Delta \theta\|_{0}^{2}=\sum_{\alpha \in I_{3}} \theta_{\alpha}^{2} A_{\alpha}^{4}$ for all $\theta \in W$. 


\section{A priori error estimates}

For a fixed number $N \geq 2$, define the finite dimensional subspaces $V_{N}$ and $W_{N}$ of $V$ and $W$ by:

$$
V_{N} \equiv\left\{\mathbf{u} \in V: \xi_{\alpha}=\eta_{\alpha}=0, \text { if }|\alpha| \equiv \alpha_{1}+\alpha_{2}+\alpha_{3}>N\right\}, \quad W_{N} \equiv\left\{\theta \in W: \theta_{\alpha}=0, \text { if }|\alpha|>N\right\} .
$$

Set $X \equiv V \times W$ and $X_{N} \equiv V_{N} \times W_{N}$. Define the projections $P_{N}: V \rightarrow V_{N}$ and $Q_{N}: W \rightarrow W_{N}$ as in [7]:

$$
\left\langle\nabla\left(\mathbf{u}-P_{N} \mathbf{u}\right), \nabla \mathbf{v}\right\rangle=0,{ }^{\forall} \mathbf{v} \in V_{N}, \quad\left\langle\nabla\left(\theta-Q_{N} \theta\right), \nabla \vartheta\right\rangle=0,{ }^{\forall} \vartheta \in W_{N},
$$

with respect to the inner product $\langle\cdot, \cdot\rangle$ on $L^{2}(\Omega)$ or properly extended spaces. Due to orthogonal relations of base functions in $X$, these projections $P_{N}$ and $Q_{N}$ are truncation operators:

$$
P_{N} \mathbf{u}=\sum_{\alpha \in I_{0, N} \equiv I_{0} \cap I_{I}, N}\left\{\xi_{\alpha} \Phi^{\alpha}+\eta_{\alpha} \Psi^{\alpha}\right\}, \quad Q_{N} \theta=\sum_{\alpha \in I_{3, N} \equiv I_{3} \cap I_{, N}} \theta_{\alpha} \phi_{3}^{\alpha},
$$

where $I_{, N} \equiv\left\{\alpha \in \mathbb{Z}_{0}^{3}:|\alpha| \leq N\right\}$. The sum $|\alpha|$ of multi-index $\alpha \in \mathbb{Z}_{0}^{3}$ can be considered as the result of the inner product between two vectors $\left[a^{-1}, b^{-1}, 1\right]$ and $\left[a \alpha_{1}, b \alpha_{2}, \alpha_{3}\right]$. This consideration with the CauchySchwartz inequality gives us

$$
|\alpha| \leq\left\|\left[a^{-1}, b^{-1}, 1\right]\right\| \cdot\left\|\left[a \alpha_{1}, b \alpha_{2}, \alpha_{3}\right]\right\|=C_{0} A_{\alpha}, \quad C_{0} \equiv \sqrt{a^{-2}+b^{-2}+1} .
$$

Note that $C_{0}$ depends only on the wave numbers $a$ and $b$, so we can say that it depends only on $\Omega$. From these characterization of projections and special estimation (6), we have

Theorem 1 For any $(\mathbf{u}, \theta) \in X$ and $\left(P_{N} \mathbf{u}, Q_{N} \theta\right) \in X_{N}$ in (5), the following holds:

$$
\begin{aligned}
\left\|\mathbf{u}-P_{N} \mathbf{u}\right\|_{0} & \leq \frac{C_{0}^{2}}{(N+1)^{2}}\|\Delta \mathbf{u}\|_{0}, \quad\left\|\nabla\left(\mathbf{u}-P_{N} \mathbf{u}\right)\right\|_{0} \leq \frac{C_{0}}{N+1}\|\Delta \mathbf{u}\|_{0}, \\
\left\|\theta-Q_{N} \theta\right\|_{0} & \leq \frac{C_{0}^{2}}{(N+1)^{2}}\|\Delta \theta\|_{0}, \quad\left\|\nabla\left(\theta-Q_{N} \theta\right)\right\|_{0} \leq \frac{C_{0}}{N+1}\|\Delta \theta\|_{0} .
\end{aligned}
$$

Proof Due to (6), we have $1 \leq \frac{C_{0}}{N+1} A_{\alpha}$ if $|\alpha|>N$. Hence we can establish the following estimates:

$$
\begin{aligned}
\left\|\mathbf{u}-P_{N} \mathbf{u}\right\|_{0}^{2} & =\sum_{\alpha \in I_{0}-I_{0, N}}\left\{\xi_{\alpha}^{2}+\eta_{\alpha}^{2}\right\} \leq \frac{C_{0}^{4}}{(N+1)^{4}} \sum_{\alpha \in I_{0}-I_{0, N}}\left\{\xi_{\alpha}^{2}+\eta_{\alpha}^{2}\right\} A_{\alpha}^{4} \leq \frac{C_{0}^{4}}{(N+1)^{4}}\|\Delta \mathbf{u}\|_{0}^{2}, \\
\left\|\nabla\left(\mathbf{u}-P_{N} \mathbf{u}\right)\right\|_{0}^{2} & =\sum_{\alpha \in I_{0}-I_{0, N}}\left\{\xi_{\alpha}^{2}+\eta_{\alpha}^{2}\right\} A_{\alpha}^{2} \leq \frac{C_{0}^{2}}{(N+1)^{2}} \sum_{\alpha \in I_{0}-I_{0, N}}\left\{\xi_{\alpha}^{2}+\eta_{\alpha}^{2}\right\} A_{\alpha}^{4} \leq \frac{C_{0}^{2}}{(N+1)^{2}}\|\Delta \mathbf{u}\|_{0}^{2}, \\
\left\|\theta-P_{N} \theta\right\|_{0}^{2} & =\sum_{\alpha \in I_{3}-I_{3, N}} \theta_{\alpha}^{2} \leq \frac{C_{0}^{4}}{(N+1)^{4}} \sum_{\alpha \in I_{3}-I_{3, N}} \theta_{\alpha}^{2} A_{\alpha}^{4} \leq \frac{C_{0}^{4}}{(N+1)^{4}}\|\Delta \theta\|_{0}^{2}, \\
\left\|\nabla\left(\theta-P_{N} \theta\right)\right\|_{0}^{2} & =\sum_{\alpha \in I_{3}-I_{3, N}} \theta_{\alpha}^{2} A_{\alpha}^{2} \leq \frac{C_{0}^{2}}{(N+1)^{2}} \sum_{\alpha \in I_{3}-I_{3, N}} \theta_{\alpha}^{2} A_{\alpha}^{4} \leq \frac{C_{0}^{2}}{(N+1)^{2}}\|\Delta \theta\|_{0}^{2} .
\end{aligned}
$$

These lead (7) after taking square root of them.

As usual, the $L^{\infty}$ norms $\|\mathbf{u}\|_{\infty}$ and $\|\theta\|_{\infty}$ of $\mathbf{u} \in V$ and $\theta \in W$ are defined by

$$
\begin{aligned}
& \|\mathbf{u}\|_{\infty} \equiv \sup _{\mathbf{x} \in \Omega}|\mathbf{u}(\mathbf{x})|, \quad\|\theta\|_{\infty} \equiv \sup _{\mathbf{x} \in \Omega}|\theta(\mathbf{x})|, \quad|\mathbf{u}|=\sqrt{u^{2}+v^{2}+w^{2}}, \quad|\theta|=\sqrt{\theta^{2}}, \\
& \mathbf{u}=[u, v, w], \quad u=\sum_{\alpha \in I_{0}} u_{\alpha} \phi_{1}^{\alpha}, \quad v=\sum_{\alpha \in I_{0}} v_{\alpha} \phi_{2}^{\alpha}, \quad w=\sum_{\alpha \in I_{0}} w_{\alpha} \phi_{3}^{\alpha}, \quad \theta=\sum_{\alpha \in I_{3}} \theta_{\alpha} \phi_{3}^{\alpha} .
\end{aligned}
$$

For a fixed $\alpha \in I_{0}$, the vector $\xi_{\alpha} \Phi^{\alpha}+\eta_{\alpha} \Psi^{\alpha}$ can be written of the form: $\left[u_{\alpha} \phi_{1}^{\alpha}, v_{\alpha} \phi_{2}^{\alpha}, w_{\alpha} \phi_{3}^{\alpha}\right]$ with

$$
u_{\alpha} \equiv-\frac{a \alpha_{1} \alpha_{3}}{A_{\alpha} B_{\alpha}} \xi_{\alpha}+\frac{b \alpha_{2}}{B_{\alpha}} \eta_{\alpha}, \quad v_{\alpha} \equiv-\frac{b \alpha_{2} \alpha_{3}}{A_{\alpha} B_{\alpha}} \xi_{\alpha}-\frac{a \alpha_{1}}{B_{\alpha}} \eta_{\alpha}, \quad w_{\alpha} \equiv \frac{B_{\alpha}}{A_{\alpha}} \xi_{\alpha}, \quad u_{\alpha}^{2}+v_{\alpha}^{2}+w_{\alpha}^{2}=\xi_{\alpha}^{2}+\eta_{\alpha}^{2} .
$$


Now, the square sum $f_{\alpha} \equiv\left(u_{\alpha} \phi_{1}^{\alpha}\right)^{2}+\left(v_{\alpha} \phi_{2}^{\alpha}\right)^{2}+\left(w_{\alpha} \phi_{1}^{\alpha}\right)^{2}$ can be bounded on $\Omega$ as follows, setting $f_{1} \equiv \cos ^{2}\left(a \alpha_{1} x\right), f_{2} \equiv \cos ^{2}\left(b \alpha_{2} y\right), f_{3} \equiv \cos ^{2}\left(\alpha_{3} z\right)$,

$$
\begin{aligned}
f_{\alpha} & =K_{\alpha}^{2}\left[u_{\alpha}^{2}\left(1-f_{1}\right) f_{2} f_{3}+v_{\alpha}^{2} f_{1}\left(1-f_{2}\right) f_{3}+w_{\alpha}^{2} f_{1} f_{2}\left(1-f_{3}\right)\right] \\
& \leq K_{\alpha}^{2}\left[u_{\alpha}^{2}+v_{\alpha}^{2}+w_{\alpha}^{2}\right]\left[\left(1-f_{1}\right) f_{2} f_{3}+f_{1}\left(1-f_{2}\right) f_{3}+f_{1} f_{2}\left(1-f_{3}\right)\right] \leq K_{\alpha}^{2}\left[\xi_{\alpha}^{2}+\eta_{\alpha}^{2}\right],
\end{aligned}
$$

since, for each $i, f_{i}$ on $\Omega$ takes value in the interval $[0,1]$. Thus, we have $\left\|\xi_{\alpha} \Phi^{\alpha}+\eta_{\alpha} \Psi^{\alpha}\right\|_{\infty} \leq K_{\alpha} \sqrt{\xi_{\alpha}^{2}+\eta_{\alpha}^{2}}$ for any $\alpha \in I_{0}$.

Lemma 2 For any $(\mathbf{u}, \theta) \in X$, it holds that

$$
\begin{aligned}
\|\mathbf{u}\|_{\infty} & \leq \frac{\pi}{3} \sqrt{6-\frac{2 \pi^{2}}{5}} C_{1}\|\Delta \mathbf{u}\|_{0}<1.50015 C_{1}\|\Delta \mathbf{u}\|_{0}, \\
\|\theta\|_{\infty} & \leq \frac{\pi}{3} \sqrt{6-\frac{36 \zeta(3)}{\pi^{2}}+\frac{\pi^{2}}{5}} C_{1}\|\Delta \theta\|_{0}<1.98398 C_{1}\|\Delta \theta\|_{0},
\end{aligned}
$$

where $C_{1} \equiv C_{0}^{2}|\Omega|^{-\frac{1}{2}}$ depends only on $\Omega$ and $\zeta(s) \equiv \sum_{n=1}^{\infty} \frac{1}{n^{s}}$ is the Riemann zeta function for $s>1$.

Proof From the above argument, $\|\mathbf{u}\|_{\infty} \leq \sum_{\alpha \in I_{0}}\left\|\xi_{\alpha} \Phi^{\alpha}+\eta_{\alpha} \Psi^{\alpha}\right\|_{\infty} \leq \sum_{\alpha \in I_{0}} K_{\alpha} \sqrt{\xi_{\alpha}^{2}+\eta_{\alpha}^{2}}$. And the CauchySchwartz inequality shows that

$$
\|\mathbf{u}\|_{\infty} \leq \sum_{\alpha \in I_{0}} K_{\alpha} \sqrt{\xi_{\alpha}^{2}+\eta_{\alpha}^{2}} \leq \sqrt{\sum_{\alpha \in I_{0}} K_{\alpha}^{2} A_{\alpha}^{-4}} \sqrt{\sum_{\alpha \in I_{0}}\left\{\xi_{\alpha}^{2}+\eta_{\alpha}^{2}\right\} A_{\alpha}^{4}} \leq \sqrt{C}\|\Delta \mathbf{u}\|_{0}, \quad C \equiv \sum_{\alpha \in I_{0}} K_{\alpha}^{2} A_{\alpha}^{-4} .
$$

The indices subset $I_{0}$ can be decomposed into four mutually disjoint subsets:

$$
\begin{array}{ll}
I_{0}^{1,1,0} \equiv[1,1,0]+\mathbb{Z}_{0}^{2} \times\{0\}, & I_{0}^{1,0,1} \equiv[1,0,1]+\mathbb{Z}_{0} \times\{0\} \times \mathbb{Z}_{0}, \\
I_{0}^{0,1,1} \equiv[0,1,1]+\{0\} \times \mathbb{Z}_{0}^{2}, & I_{0}^{1,1,1} \equiv[1,1,1]+\mathbb{Z}_{0}^{3} .
\end{array}
$$

And the numbers $n(k)$ of non-negative integer solutions of $|\alpha|=k$ and the values $K_{\alpha}^{2}$ on these subsets are:

$$
\left.n(k)\right|_{I_{0}-I_{0}^{1,1,1}}=k-1,\left.\quad n(k)\right|_{I_{0}^{1,1,1}}=\frac{(k-1)(k-2)}{2},\left.\quad K_{\alpha}^{2}\right|_{I_{0}-I_{0}^{1,1,1}}=\frac{4}{|\Omega|},\left.\quad K_{\alpha}^{2}\right|_{I_{0}^{1,1,1}}=\frac{8}{|\Omega|} .
$$

Due to (6) and the above relations, we can bound $C$ as follows:

$$
\begin{aligned}
C & \leq C_{0}^{4} \sum_{\alpha \in I_{0}} K_{\alpha}^{2}|\alpha|^{-4}=\frac{4 C_{0}^{4}}{|\Omega|}\left[3 \sum_{k=2}^{\infty} \frac{k-1}{k^{4}}+2 \sum_{k=3}^{\infty} \frac{(k-1)(k-2)}{2 k^{4}}\right]=4 C_{1}^{2} \sum_{k=1}^{\infty} \frac{(k-1)(k+1)}{k^{4}} \\
& =4 C_{1}^{2} \sum_{k=1}^{\infty}\left[\frac{1}{k^{2}}-\frac{1}{k^{4}}\right]=4 C_{1}^{2}\left[\frac{\pi^{2}}{6}-\frac{\pi^{4}}{90}\right]=\frac{\pi^{2}}{9}\left[6-\frac{2 \pi^{2}}{5}\right] C_{1}^{2},
\end{aligned}
$$

which proves the first part of the lemma.

Next, taking account that $\|\theta\|_{\infty} \leq \sum_{\alpha \in I_{3}}\left\|\theta_{\alpha} \phi_{3}^{\alpha}\right\|_{\infty}=\sum_{\alpha \in I_{3}} K_{\alpha}\left|\theta_{\alpha}\right|$, similar to the above, we have

$$
\|\theta\|_{\infty} \leq \sum_{\alpha \in I_{3}} K_{\alpha}\left|\theta_{\alpha}\right| \leq \sqrt{\sum_{\alpha \in I_{3}} K_{\alpha}^{2} A_{\alpha}^{-4}} \sqrt{\sum_{\alpha \in I_{3}} \theta_{\alpha}^{2} A_{\alpha}^{4}} \leq \sqrt{\tilde{C}}\|\Delta \theta\|_{0}, \quad \text { with } \quad \tilde{C} \equiv \sum_{\alpha \in I_{3}} K_{\alpha}^{2} A_{\alpha}^{-4} .
$$

The indices subset $I_{3}$ can be decomposed into four mutually disjoint subsets:

$$
I_{3}^{0,0,1} \equiv[0,0,1]+\{0\}^{2} \times \mathbb{Z}_{0}, I_{3}^{1,0,1} \equiv I_{0}^{1,0,1}, I_{3}^{0,1,1} \equiv I_{0}^{0,1,1}, I_{3}^{1,1,1} \equiv I_{0}^{1,1,1} .
$$

And $n(k)$ and $K_{\alpha}^{2}$ on these subsets are:

$$
\begin{aligned}
& \left.n(k)\right|_{I_{3}^{0,0,1}}=1,\left.\quad n(k)\right|_{I_{3}^{1,0,1} \cup I_{3}^{0,1,1}}=k-1,\left.\quad n(k)\right|_{I_{3}^{1,1,1}}=\frac{(k-1)(k-2)}{2}, \\
& \left.K_{\alpha}^{2}\right|_{I_{3}^{0,0,1}}=\frac{2}{|\Omega|},\left.\quad K_{\alpha}^{2}\right|_{I_{3}^{1,0,1} \cup I_{3}^{0,1,1}}=\frac{4}{|\Omega|},\left.\quad K_{\alpha}^{2}\right|_{I_{3}^{1,1,1}}=\frac{8}{|\Omega|} .
\end{aligned}
$$


Thus, the bound $\tilde{C}$ can be obtained as follows:

$$
\begin{aligned}
\tilde{C} & \leq C_{0}^{4} \sum_{\alpha \in I_{3}} K_{\alpha}^{2}|\alpha|^{-4}=\frac{2 C_{0}^{4}}{|\Omega|}\left[\sum_{k=1}^{\infty} \frac{1}{k^{4}}+4 \sum_{k=2}^{\infty} \frac{k-1}{k^{4}}+4 \sum_{k=3}^{\infty} \frac{(k-1)(k-2)}{2 k^{4}}\right]=2 C_{1}^{2} \sum_{k=1}^{\infty} \frac{2 k^{2}-2 k+1}{k^{4}} \\
& =2 C_{1}^{2} \sum_{k=1}^{\infty}\left[\frac{2}{k^{2}}-\frac{2}{k^{3}}+\frac{1}{k^{4}}\right]=2 C_{1}^{2}\left[\frac{\pi^{2}}{3}-2 \zeta(3)+\frac{\pi^{4}}{90}\right]=\frac{\pi^{2}}{9}\left[6-\frac{36 \zeta(3)}{\pi^{2}}+\frac{\pi^{2}}{5}\right] C_{1}^{2},
\end{aligned}
$$

which completes the proof.

Since $\Omega$ is a rectangle with side lengths $\frac{2 \pi}{a}, \frac{2 \pi}{b}$, and $\pi$, we can also use the result in [6] as follows $(0<a, b \leq 1)$ :

$$
\begin{aligned}
\|\theta\|_{\infty} & \leq|\Omega|^{-\frac{1}{2}}\left[\gamma_{0}\|\theta\|_{0}+\frac{\gamma_{1} \pi c_{1}}{\sqrt{3}}\|\nabla \theta\|_{0}+\frac{\gamma_{2} \pi^{2} c_{2}}{3}\left\|\nabla^{2} \theta\right\|_{0}\right], \\
c_{1} & =\sqrt{4\left(a^{-2}+b^{-2}\right)+1} \geq \sqrt{3} C_{0}, \\
c_{2} & =\sqrt{c_{1}^{4}+\frac{4}{5}\left[16\left(a^{-4}+b^{-4}\right)+1\right]} \geq \sqrt{\frac{19}{15}} c_{1}^{2} \geq 3 \sqrt{\frac{19}{15}} C_{0}^{2}, \\
\frac{\gamma_{2} \pi^{2} c_{2}}{3} & \geq 5.59879 \gamma_{2} \frac{\pi}{3} \sqrt{6-\frac{36 \zeta(3)}{\pi^{2}}+\frac{\pi^{2}}{5}} C_{0}^{2} \geq 2.31862 \frac{\pi}{3} \sqrt{6-\frac{36 \zeta(3)}{\pi^{2}}+\frac{\pi^{2}}{5}} C_{0}^{2} .
\end{aligned}
$$

Here, we have used the fact taht $\gamma_{2}$ is estimated as 0.41413 in [6]. Thus the coefficients in Lemma 2 are at least twice finer than those in [6].

Corollary 3 Under the same assumptions of Theorem 1, the following holds for the same constant $C_{1}$ in Lemma 2:

$$
\begin{gathered}
\left\|\mathbf{u}-P_{N} \mathbf{u}\right\|_{\infty} \leq 2 C_{1} \sqrt{\frac{1}{N}-\frac{1}{3(N+1)^{3}}}\|\Delta \mathbf{u}\|_{0}<\frac{2 C_{1}}{\sqrt{N}}\|\Delta \mathbf{u}\|_{0}, \\
\left\|\theta-Q_{N} \theta\right\|_{\infty} \leq 2 C_{1} \sqrt{\frac{1}{N}-\frac{1}{2(N+1)^{2}}+\frac{1}{6 N^{3}}}\|\Delta \theta\|_{0}<\frac{2 C_{1}}{\sqrt{N}}\|\Delta \theta\|_{0} .
\end{gathered}
$$

Proof We can proceed as that of Lemma 2 and need to change $C$ for each case. For $\left\|\mathbf{u}-P_{N} \mathbf{u}\right\|_{\infty}$,

$$
\begin{aligned}
C & \equiv \sum_{\alpha \in I_{0}-I_{0, N}} K_{\alpha}^{2} A_{\alpha}^{-4} \leq C_{0}^{4} \sum_{\alpha \in I_{0}-I_{0, N}} K_{\alpha}^{2}|\alpha|^{-4}=4 C_{1}^{2}\left[3 \sum_{k=N+1}^{\infty} \frac{k-1}{k^{4}}+2 \sum_{k=N+1}^{\infty} \frac{(k-1)(k-2)}{2 k^{4}}\right] \\
& =4 C_{1}^{2} \sum_{k=N+1}^{\infty} \frac{(k-1)(k+1)}{k^{4}}=4 C_{1}^{2} \sum_{k=N+1}^{\infty}\left[\frac{1}{k^{2}}-\frac{1}{k^{4}}\right]<4 C_{1}^{2}\left[\int_{N}^{\infty} \frac{1}{x^{2}} d x-\int_{N+1}^{\infty} \frac{1}{x^{4}} d x\right] \\
& =4\left[\frac{1}{N}-\frac{1}{3(N+1)^{3}}\right] C_{1}^{2}<\frac{4}{N} C_{1}^{2} .
\end{aligned}
$$

Similarly, we have the following estimates for $\left\|\theta-Q_{N} \theta\right\|_{\infty}$ :

$$
\begin{aligned}
\tilde{C} & \equiv \sum_{\alpha \in I_{3}-I_{3, N}} K_{\alpha}^{2} A_{\alpha}^{-4} \leq C_{0}^{4} \sum_{\alpha \in I_{3}-I_{3, N}} K_{\alpha}^{2}|\alpha|^{-4}=2 C_{1}^{2}\left[\sum_{k=N+1}^{\infty} \frac{1}{k^{4}}+4 \sum_{k=N+1}^{\infty} \frac{k-1}{k^{4}}+4 \sum_{k=N+1}^{\infty} \frac{(k-1)(k-2)}{2 k^{4}}\right] \\
& =2 C_{1}^{2} \sum_{k=N+1}^{\infty} \frac{2 k^{2}-2 k+1}{k^{4}}=2 C_{1}^{2} \sum_{k=N+1}^{\infty}\left[\frac{2}{k^{2}}-\frac{2}{k^{3}}+\frac{1}{k^{4}}\right]<2 C_{1}^{2}\left[\int_{N}^{\infty} \frac{2}{x^{2}} d x-\int_{N+1}^{\infty} \frac{2}{x^{3}} d x+\int_{N}^{\infty} \frac{1}{x^{4}} d x\right] \\
& =2\left[\frac{2}{N}-\frac{1}{(N+1)^{2}}+\frac{1}{3 N^{3}}\right] C_{1}^{2}=4\left[\frac{1}{N}-\frac{1}{2(N+1)^{2}}+\frac{1}{6 N^{3}}\right] C_{1}^{2}<\frac{4}{N} C_{1}^{2} .
\end{aligned}
$$

The last inequality holds due to the assumption $N \geq 2$. 


\section{A fixed point formulation}

The steady state solution of (3) can be written of the form:

$$
\begin{aligned}
-\Delta \mathbf{u}+\nabla p & =\mathbf{f}(\mathbf{u}, \theta), \\
\nabla \cdot \mathbf{u} & =0, \\
-\Delta \theta & =g(\mathbf{u}, \theta),
\end{aligned}
$$

where the right hand sides of (9) are defined by

$$
\mathbf{f}(\mathbf{u}, \theta)=-\frac{1}{\mathscr{P}}(\mathbf{u} \cdot \nabla) \mathbf{u}+\mathscr{R} \theta \mathbf{e}_{z}, \quad g(\mathbf{u}, \boldsymbol{\theta})=-(\mathbf{u} \cdot \nabla) \theta+w .
$$

If $\mathbf{u} \in V$, then $P_{N} \mathbf{u}$ satisfies (9b) and $-\Delta P_{N} \mathbf{u}$ converges to $-\Delta \mathbf{u}$ in $L^{2}$ sense. Taking inner product for both sides of (9a) with $-\Delta P_{N} \mathbf{u}$, we have

$$
\begin{aligned}
\left\langle-\Delta \mathbf{u},-\Delta P_{N} \mathbf{u}\right\rangle & =\left\langle-\Delta \mathbf{u},-\Delta P_{N} \mathbf{u}\right\rangle+\left\langle p, \Delta \nabla \cdot P_{N} \mathbf{u}\right\rangle=\left\langle-\Delta \mathbf{u},-\Delta P_{N} \mathbf{u}\right\rangle+\left\langle p, \nabla \cdot \Delta P_{N} \mathbf{u}\right\rangle \\
& =\left\langle-\Delta \mathbf{u},-\Delta P_{N} \mathbf{u}\right\rangle+\left\langle\nabla p,-\Delta P_{N} \mathbf{u}\right\rangle=\left\langle\mathbf{f},-\Delta P_{N} \mathbf{u}\right\rangle .
\end{aligned}
$$

This identity converges to $\langle-\Delta \mathbf{u},-\Delta \mathbf{u}\rangle=\langle\mathbf{f},-\Delta \mathbf{u}\rangle$ which means $\|\Delta \mathbf{u}\|_{0} \leq\|\mathbf{f}\|_{0}$. And taking inner product for both sides of (9c) with $-\Delta \theta$, we have $\langle-\Delta \theta,-\Delta \theta\rangle=\langle g,-\Delta \theta\rangle$ and $\|\Delta \theta\|_{0} \leq\|g\|_{0}$.

Now, setting $F(\mathbf{u}, \theta) \equiv(\mathbf{f}(\mathbf{u}, \theta), g(\mathbf{u}, \theta))$, the weak form of (9) is written as :

$$
\langle\nabla(\mathbf{u}, \theta), \nabla(\mathbf{v}, \vartheta)\rangle=\langle F(\mathbf{u}, \theta),(\mathbf{v}, \vartheta)\rangle, \quad{ }^{\forall}(\mathbf{v}, \vartheta) \in X
$$

We call the solution operator $\mathscr{S}$ for (9) as Stokes operator. Thus $(\mathbf{u}, \theta)=\mathscr{S} F(\mathbf{u}, \theta)$ means

$$
\langle\nabla \mathscr{S} F(\mathbf{u}, \theta), \nabla(\mathbf{v}, \vartheta)\rangle=\langle F(\mathbf{u}, \theta),(\mathbf{v}, \vartheta)\rangle, \quad{ }^{\forall}(\mathbf{v}, \vartheta) \in X
$$

Note that we always have $\mathscr{S}^{-1}(\mathbf{u}, \theta)=(-\Delta \mathbf{u}+\nabla p,-\Delta \theta)$ with an associated pressure $p=p(\mathbf{u}, \theta)$.

Usually, we use Newton's method (see [4]) to get an approximate solution $\left(\mathbf{u}_{N}, \theta_{N}\right) \in X_{N}$ of (9) and define the approximate pressure $p_{N}$ by

$$
\nabla p_{N} \equiv \mathbf{f}_{N}\left(\mathbf{u}_{N}, \theta_{N}\right)+\Delta \mathbf{u}_{N}
$$

where $\mathbf{f}_{N}$ is the truncation up to $I_{, N}$ of the expansion of $\mathbf{f}$. For the solution $(\mathbf{u}, \theta)$ of (9) with its associated pressure $p$, let $(\overline{\mathbf{u}}, \bar{\theta}) \equiv\left(\mathbf{u}-\mathbf{u}_{N}, \theta-\theta_{N}\right)$ and $\bar{p} \equiv p-p_{N}$. Then we have the following residual equations:

$$
\begin{aligned}
-\Delta \overline{\mathbf{u}}+\nabla \bar{p} & =\mathbf{f}\left(\mathbf{u}_{N}+\overline{\mathbf{u}}, \theta_{N}+\bar{\theta}\right)+\Delta \mathbf{u}_{N}-\nabla p_{N}, \\
\nabla \cdot \overline{\mathbf{u}} & =0, \\
-\Delta \bar{\theta} & =g\left(\mathbf{u}_{N}+\overline{\mathbf{u}}, \theta_{N}+\bar{\theta}\right)+\Delta \theta_{N},
\end{aligned}
$$

$\operatorname{Set} \bar{F}(\overline{\mathbf{u}}, \bar{\theta}) \equiv\left(\mathbf{f}\left(\mathbf{u}_{N}+\overline{\mathbf{u}}, \theta_{N}+\bar{\theta}\right)+\Delta \mathbf{u}_{N}-\nabla p_{N}, g\left(\mathbf{u}_{N}+\overline{\mathbf{u}}, \theta_{N}+\bar{\theta}\right)+\Delta \theta_{N}\right) \equiv(\overline{\mathbf{f}}(\overline{\mathbf{u}}, \bar{\theta}), \bar{g}(\overline{\mathbf{u}}, \bar{\theta}))$, then the Stokes operator $\mathscr{S}$ gives us a fixed point problem from (11):

$$
(\overline{\mathbf{u}}, \bar{\theta})=\mathscr{S} \bar{F}(\overline{\mathbf{u}}, \bar{\theta}) \equiv \mathscr{K}(\overline{\mathbf{u}}, \bar{\theta}) .
$$

Since $X \subset H^{1}(\Omega)^{4}, \mathscr{K}$ is a compact operator on $X$. Hence by Schauder's fixed point theorem, if we find a nonempty, closed, convex, and bounded set $U \subset X$ satisfying $\mathscr{K} U \subset U$, then there exists a solution of (12) in $U$ which is called a candidate set.

Define $\mathbf{P}_{N}: X \rightarrow X_{N}$ by $\mathbf{P}_{N}=\left(P_{N}, Q_{N}\right)$, then (5) can be simplified as: for $(\mathbf{u}, \theta) \in X$

$$
\left\langle\nabla\left((\mathbf{u}, \boldsymbol{\theta})-\mathbf{P}_{N}(\mathbf{u}, \boldsymbol{\theta})\right), \nabla(\mathbf{v}, \vartheta)\right\rangle=0, \quad{ }^{\forall}(\mathbf{v}, \vartheta) \in X_{N}
$$

Then (12) can be decomposed into two parts:

$$
\begin{aligned}
\mathbf{P}_{N}(\overline{\mathbf{u}}, \bar{\theta}) & =\mathbf{P}_{N} \mathscr{K}(\overline{\mathbf{u}}, \bar{\theta}), \\
\left(I-\mathbf{P}_{N}\right)(\overline{\mathbf{u}}, \bar{\theta}) & =\left(I-\mathbf{P}_{N}\right) \mathscr{K}(\overline{\mathbf{u}}, \bar{\theta}) .
\end{aligned}
$$


The Fréchet derivative $F^{\prime}(\mathbf{u}, \theta)$ of $F$ at $(\mathbf{u}, \theta)$ has the form: for any $(\overline{\mathbf{u}}, \bar{\theta}) \in X$,

$$
\begin{aligned}
F^{\prime}(\mathbf{u}, \theta)(\overline{\mathbf{u}}, \bar{\theta}) & \equiv\left(\mathbf{f}^{\prime}(\mathbf{u}, \theta)(\overline{\mathbf{u}}, \bar{\theta}), g^{\prime}(\mathbf{u}, \theta)(\overline{\mathbf{u}}, \bar{\theta})\right) \\
\mathbf{f}^{\prime}(\mathbf{u}, \theta)(\overline{\mathbf{u}}, \bar{\theta}) & \equiv-\frac{1}{\mathscr{P}}[(\mathbf{u} \cdot \nabla) \overline{\mathbf{u}}+(\overline{\mathbf{u}} \cdot \nabla) \mathbf{u}]+\mathscr{R} \bar{\theta} \mathbf{e}_{z}, \\
g^{\prime}(\mathbf{u}, \theta)(\overline{\mathbf{u}}, \bar{\theta}) & \equiv-[(\mathbf{u} \cdot \nabla) \bar{\theta}+(\overline{\mathbf{u}} \cdot \nabla) \theta]+\bar{w}
\end{aligned}
$$

Now, define $\mathscr{L}_{N}: X_{N} \rightarrow X_{N}$ by

$$
\left.\mathscr{L}_{N} \equiv \mathbf{P}_{N}\left[I-\mathscr{S} F^{\prime}\left(\mathbf{u}_{N}, \theta_{N}\right)\right]\right|_{X_{N}},
$$

and assume $\mathscr{L}_{N}$ is regular or one-to-one and onto. And we can express $\mathscr{L}_{N}$ as:

$$
\mathscr{L}_{N}=\left.\mathbf{P}_{N} \mathscr{S}\left[\mathscr{S}^{-1}-F^{\prime}\left(\mathbf{u}_{N}, \theta_{N}\right)\right]\right|_{X_{n}}=\mathbf{P}_{N} \mathscr{S} \mathscr{L}_{0},\left.\quad \mathscr{L}_{0} \equiv\left[\mathscr{S}^{-1}-F^{\prime}\left(\mathbf{u}_{N}, \theta_{N}\right)\right]\right|_{X_{n}} .
$$

Define the Newton-like iteration operator $\mathscr{N}: X \rightarrow X_{N}$ for (12) and the new map $\mathscr{T}$ as follows:

$$
\mathscr{N} \equiv \mathbf{P}_{N}-\mathscr{L}_{N}^{-1} \mathbf{P}_{N}(I-\mathscr{K}), \quad \mathscr{T} \equiv \mathscr{N}+\left(I-\mathbf{P}_{N}\right) \mathscr{K}
$$

The second part of $\mathscr{T}$ is expected to be small or contractive if the truncation number $N$ is sufficiently large. The operator $\mathscr{N}$ is also compact since it maps $X$ into the finite dimensional space $X_{N}$, and so is $\mathscr{T}$.

Lemma 4 The problem (14) is equivalent to the following fixed point problem:

$$
(\overline{\mathbf{u}}, \bar{\theta})=\mathscr{T}(\overline{\mathbf{u}}, \bar{\theta}) \text {. }
$$

Proof Assume $(\overline{\mathbf{u}}, \bar{\theta}) \in X$ satisfies (14), then $\mathscr{N}(\overline{\mathbf{u}}, \bar{\theta})=\mathbf{P}_{N}(\overline{\mathbf{u}}, \bar{\theta})$ which means

$$
\mathscr{T}(\overline{\mathbf{u}}, \bar{\theta})=\mathbf{P}_{N}(\overline{\mathbf{u}}, \bar{\theta})+\left(I-\mathbf{P}_{N}\right) \mathscr{K}(\overline{\mathbf{u}}, \bar{\theta})=\mathbf{P}_{N}(\overline{\mathbf{u}}, \bar{\theta})+\left(I-\mathbf{P}_{N}\right)(\overline{\mathbf{u}}, \bar{\theta})=(\overline{\mathbf{u}}, \bar{\theta}) .
$$

Thus $(\overline{\mathbf{u}}, \bar{\theta})$ satisfies (15). On the other hand, if $(\overline{\mathbf{u}}, \bar{\theta})$ satisfies (15), then

$$
\begin{aligned}
\mathbf{P}_{N}(\overline{\mathbf{u}}, \bar{\theta}) & =\mathbf{P}_{N} \mathscr{T}(\overline{\mathbf{u}}, \bar{\theta})=\mathbf{P}_{N} \mathscr{N}(\overline{\mathbf{u}}, \bar{\theta})=\mathbf{P}_{N}(\overline{\mathbf{u}}, \bar{\theta})-\mathscr{L}_{N}^{-1} \mathbf{P}_{N}(I-\mathscr{K})(\overline{\mathbf{u}}, \bar{\theta}) \\
& \Rightarrow \mathscr{L}_{N}^{-1} \mathbf{P}_{N}(I-\mathscr{K})(\overline{\mathbf{u}}, \bar{\theta})=0 \Rightarrow \mathbf{P}_{N}(I-\mathscr{K})(\overline{\mathbf{u}}, \bar{\theta})=0 \Rightarrow \mathbf{P}_{N}(\overline{\mathbf{u}}, \bar{\theta})=\mathbf{P}_{N} \mathscr{K}(\overline{\mathbf{u}}, \bar{\theta}), \\
\left(I-\mathbf{P}_{N}\right)(\overline{\mathbf{u}}, \bar{\theta}) & =\left(I-\mathbf{P}_{N}\right) \mathscr{T}(\overline{\mathbf{u}}, \bar{\theta})=\left(I-\mathbf{P}_{N}\right) \mathscr{K}(\overline{\mathbf{u}}, \bar{\theta}) .
\end{aligned}
$$

Here the second implication is due to the assumption on the regularity of $\mathscr{L}_{N}$.

From Lemma 4, we have an alternative verification condition of the form: $\mathscr{T} U \subset U$ for a candidate set $U$ which is nonempty, closed, convex, and bounded in $X$.

Now, for given real numbers $\bar{\xi}_{\alpha}, \bar{\eta}_{\alpha}, \bar{\theta}_{\alpha} \geq 0$, set real intervals as $\left[\bar{\xi}_{\alpha}\right] \equiv\left[-\bar{\xi}_{\alpha}, \bar{\xi}_{\alpha}\right],\left[\bar{\eta}_{\alpha}\right] \equiv\left[-\bar{\eta}_{\alpha}, \bar{\eta}_{\alpha}\right]$, $\left[\bar{\theta}_{\alpha}\right] \equiv\left[-\bar{\theta}_{\alpha}, \bar{\theta}_{\alpha}\right]$, and define

$$
U_{N} \equiv\left\{\left(\sum_{\alpha \in I_{0, N}}\left\{\xi_{\alpha} \Phi^{\alpha}+\eta_{\alpha} \Psi^{\alpha}\right\}, \sum_{\alpha \in I_{3, N}} \theta_{\alpha} \phi_{3}^{\alpha}\right) \in X_{N}: \xi_{\alpha} \in\left[\bar{\xi}_{\alpha}\right], \eta_{\alpha} \in\left[\bar{\eta}_{\alpha}\right], \theta_{\alpha} \in\left[\bar{\theta}_{\alpha}\right]\right\} .
$$

And for given $m_{1}, m_{2} \geq 0$, we define

$$
U_{*} \equiv\left\{\begin{array}{ll}
(\mathbf{u}, \theta) \in X_{N}^{\perp}: & \|\mathbf{u}\|_{0} \leq \frac{C_{0}^{2}}{(N+1)^{2}} m_{1},\|\nabla \mathbf{u}\|_{0} \leq \frac{C_{0}}{N+1} m_{1},\|\mathbf{u}\|_{\infty} \leq \frac{2 C_{1}}{\sqrt{N}} m_{1}, \\
\|\theta\|_{0} \leq \frac{C_{0}^{2}}{(N+1)^{2}} m_{2},\|\nabla \theta\|_{0} \leq \frac{C_{0}}{N+1} m_{2}
\end{array}\right\} .
$$

Here $X_{N}^{\perp}$ is the orthogonal complement of $X_{N}$ in $X$ with respect to the projection $\mathbf{P}_{N}$ defined by (13). Now, set $U \equiv U_{N} \oplus U_{*}$, then we obtain:

Theorem 5 Let $U_{N}, U_{*}$ and $U$ be sets defined as above. If

$$
\begin{aligned}
& \mathscr{N} U \subset U_{N}, \\
&\left(I-\mathbf{P}_{N}\right) \mathscr{K} U \subset U_{*} .
\end{aligned}
$$

then there exists a fixed point of $\mathscr{T}$ in $U$.

Proof Clearly, $(\mathbf{0}, 0) \in U$ which means $U$ is non-empty. Due to the definition, $U$ is closed, convex and bounded in $X$. Under the condition (18), we have

$$
\mathscr{T} U \subset \mathscr{N} U+\left(I-\mathbf{P}_{N}\right) \mathscr{K} U \subset U_{N}+U_{*}=U .
$$

Since $\mathscr{T}$ is compact, there exists a fixed point of $\mathscr{T}$ in $U$ by Schauder's fixed point theorem. 


\section{Computable verification conditions}

To construct the candidate set $U$ in $X$ satisfying (18), we use an algorithm based on iterative scheme as in [7].

First, set the initial values $\bar{\xi}_{\alpha}^{(0)}=\bar{\eta}_{\alpha}^{(0)}=\bar{\theta}_{\alpha}^{(0)}=0$, and $m_{1}^{(0)}=m_{2}^{(0)}=0$, which means $U^{(0)}$ contains only one element $(\mathbf{0}, 0)$. For $k \geq 0$, with a fixed inflation factor $0<\delta \ll 1$, set

$$
\bar{\xi}_{\alpha}^{\left(k+\frac{1}{2}\right)}=\bar{\xi}_{\alpha}^{(k)}(1+\delta), \quad \bar{\eta}_{\alpha}^{\left(k+\frac{1}{2}\right)}=\bar{\eta}_{\alpha}^{(k)}(1+\delta), \quad \bar{\theta}_{\alpha}^{\left(k+\frac{1}{2}\right)}=\bar{\theta}_{\alpha}^{(k)}(1+\delta), \quad m_{i}^{\left(k+\frac{1}{2}\right)}=m_{i}^{(k)}(1+\delta), i=1,2,
$$

which define $\delta$-inflations $U_{N}^{\left(k+\frac{1}{2}\right)}$ and $U_{*}^{\left(k+\frac{1}{2}\right)}$ of $U_{N}^{(k)}$ and $U_{*}^{(k)}$ respectively. Set the $\delta$-inflation $U^{\left(k+\frac{1}{2}\right)}$ of $U^{(k)}$ as the direct sum of $U_{N}^{\left(k+\frac{1}{2}\right)}$ and $U_{*}^{\left(k+\frac{1}{2}\right)}$, i.e., $U^{\left(k+\frac{1}{2}\right)} \equiv U_{N}^{\left(k+\frac{1}{2}\right)} \oplus U_{*}^{\left(k+\frac{1}{2}\right)}$. Now, $U^{(k+1)}$ can be constructed as the direct sum of $U_{N}^{(k+1)}$ and $U_{*}^{(k+1)}$ as follows:

$$
U_{N}^{(k+1)} \equiv \mathscr{N} U^{\left(k+\frac{1}{2}\right)}, \quad m_{1}^{(k+1)} \equiv\left\|\overline{\mathbf{f}}\left(U^{\left(k+\frac{1}{2}\right)}\right)\right\|_{0}, \quad m_{2}^{(k+1)} \equiv\left\|\bar{g}\left(U^{\left(k+\frac{1}{2}\right)}\right)\right\|_{0}
$$

where $\|f(U)\|_{0} \equiv \sup \left\{\|f(\mathbf{u}, \theta)\|_{0}:(\mathbf{u}, \theta) \in U\right\}$ for any function $f$. Note that $U^{(k+1)}$ cannot be calculated exactly, but its over-estimated enclosure can be obtained and will be set as $U_{N}^{(k+1)}$ in the actual calculation on a computer. Thus the verification condition in a computer is:

Theorem 6 For some $k$, if the following conditions

$$
\bar{\xi}_{\alpha}^{(k+1)}<\bar{\xi}_{\alpha}^{\left(k+\frac{1}{2}\right)}, \quad \bar{\eta}_{\alpha}^{(k+1)}<\bar{\eta}_{\alpha}^{\left(k+\frac{1}{2}\right)}, \quad \bar{\theta}_{\alpha}^{(k+1)}<\bar{\theta}_{\alpha}^{\left(k+\frac{1}{2}\right)}, \quad m_{i}^{(k+1)}<m_{i}^{\left(k+\frac{1}{2}\right)}, i=1,2,
$$

hold, then the set $U^{\left(k+\frac{1}{2}\right)}$ contains an element $(\overline{\mathbf{u}}, \bar{\theta})$ satisfying $(\overline{\mathbf{u}}, \bar{\theta})=\mathscr{T}(\overline{\mathbf{u}}, \bar{\theta})$.

Proof Due to Theorem 5, it is sufficient to check (18) holds for $U^{\left(k+\frac{1}{2}\right)}$. By the condition (20) and the definition (19), we have $\mathscr{N} U^{\left(k+\frac{1}{2}\right)}=U_{N}^{(k+1)} \subset U_{N}^{\left(k+\frac{1}{2}\right)}$. And for any $(\mathbf{u}, \theta) \in\left(I-\mathbf{P}_{N}\right) \mathscr{K} U^{\left(k+\frac{1}{2}\right)}$, there exists $(\overline{\mathbf{u}}, \bar{\theta}) \in U^{\left(k+\frac{1}{2}\right)}$ such that $(\mathbf{u}, \theta)=\left(I-\mathbf{P}_{N}\right) \mathscr{S} \bar{F}(\overline{\mathbf{u}}, \bar{\theta})$. Using Theorem 1, Corollary 3 and (19), we obtain

$$
\begin{aligned}
\|\mathbf{u}\|_{0} & =\left\|\left(I-P_{N}\right) \Pi_{1} \mathscr{S} \bar{F}(\overline{\mathbf{u}}, \bar{\theta})\right\|_{0} \leq \frac{C_{0}^{2}}{(N+1)^{2}}\|\overline{\mathbf{f}}(\overline{\mathbf{u}}, \bar{\theta})\|_{0} \leq \frac{C_{0}^{2}}{(N+1)^{2}} m_{1}^{(k+1)}<\frac{C_{0}^{2}}{(N+1)^{2}} m_{1}^{\left(k+\frac{1}{2}\right)}, \\
\|\nabla \mathbf{u}\|_{0} & =\left\|\nabla\left(I-P_{N}\right) \Pi_{1} \mathscr{S} \bar{F}(\overline{\mathbf{u}}, \bar{\theta})\right\|_{0} \leq \frac{C_{0}}{N+1}\|\overline{\mathbf{f}}(\overline{\mathbf{u}}, \bar{\theta})\|_{0} \leq \frac{C_{0}}{N+1} m_{1}^{(k+1)}<\frac{C_{0}}{N+1} m_{1}^{\left(k+\frac{1}{2}\right)}, \\
\|\mathbf{u}\|_{\infty} & =\left\|\left(I-P_{N}\right) \Pi_{1} \mathscr{S} \bar{F}(\overline{\mathbf{u}}, \bar{\theta})\right\|_{\infty} \leq \frac{2 C_{1}}{\sqrt{N}}\|\overline{\mathbf{f}}(\overline{\mathbf{u}}, \bar{\theta})\|_{0} \leq \frac{2 C_{1}}{\sqrt{N}} m_{1}^{(k+1)}<\frac{2 C_{1}}{\sqrt{N}} m_{1}^{\left(k+\frac{1}{2}\right)}, \\
\|\theta\|_{0} & =\left\|\left(I-Q_{N}\right) \Pi_{2} \mathscr{S} \bar{F}(\overline{\mathbf{u}}, \bar{\theta})\right\|_{0} \leq \frac{C_{0}^{2}}{(N+1)^{2}}\|\bar{g}(\overline{\mathbf{u}}, \bar{\theta})\|_{0} \leq \frac{C_{0}^{2}}{(N+1)^{2}} m_{2}^{(k+1)}<\frac{C_{0}^{2}}{(N+1)^{2}} m_{2}^{\left(k+\frac{1}{2}\right)}, \\
\|\nabla \theta\|_{0} & =\left\|\nabla\left(I-Q_{N}\right) \Pi_{2} \mathscr{S} \bar{F}(\overline{\mathbf{u}}, \bar{\theta})\right\|_{0} \leq \frac{C_{0}}{N+1}\|\bar{g}(\overline{\mathbf{u}}, \bar{\theta})\|_{0} \leq \frac{C_{0}}{N+1} m_{2}^{(k+1)}<\frac{C_{0}}{N+1} m_{2}^{\left(k+\frac{1}{2}\right)},
\end{aligned}
$$

where $\Pi_{1}: X \rightarrow V$ and $\Pi_{2}: X \rightarrow W$ are the natural projections from $X$ to $V$ and $W$, respectively. These estimates mean that $(\mathbf{u}, \theta) \in U_{*}^{\left(k+\frac{1}{2}\right)}$ and $\left(I-\mathbf{P}_{N}\right) \mathscr{K} U^{\left(k+\frac{1}{2}\right)} \subset U_{*}^{\left(k+\frac{1}{2}\right)}$ holds.

To determine the finite dimensional set $U_{N}^{(k+1)}$ in (19), we need to compute $\mathscr{N}$ on $U$. At first, from definitions of $\mathscr{L}_{N}$ and $\mathscr{K}$, we can rewrite $\mathscr{N}$ as follows:

$$
\mathscr{N}=\mathscr{L}_{N}^{-1}\left[\mathscr{L}_{N} \mathbf{P}_{N}-\mathbf{P}_{N}+\mathbf{P}_{N} \mathscr{K}\right]=\mathscr{L}_{N}^{-1} \mathbf{P}_{N} \mathscr{S} F_{0}, \quad F_{0} \equiv \bar{F}-F^{\prime}\left(\mathbf{u}_{N}, \theta_{N}\right) \mathbf{P}_{N}
$$

For any fixed $(\overline{\mathbf{u}}, \bar{\theta}) \in U$, set $\left(\mathbf{u}_{h}, \theta_{h}\right) \equiv \mathscr{N}(\overline{\mathbf{u}}, \bar{\theta})$ and operate $\mathscr{L}_{N}$ on both sides, then

$$
\mathbf{P}_{N} \mathscr{S} \mathscr{L}_{0}\left(\mathbf{u}_{h}, \theta_{h}\right)=\mathscr{L}_{N}\left(\mathbf{u}_{h}, \theta_{h}\right)=\mathbf{P}_{N} \mathscr{S} F_{0}(\overline{\mathbf{u}}, \bar{\theta})
$$

Using the projection property (13) of $\mathbf{P}_{N}$, we can derive

$$
\left\langle\nabla \mathscr{S} \mathscr{L}_{0}\left(\mathbf{u}_{h}, \theta_{h}\right), \nabla\left(\mathbf{v}_{N}, \vartheta_{N}\right)\right\rangle=\left\langle\nabla \mathscr{S} F_{0}(\overline{\mathbf{u}}, \bar{\theta}), \nabla\left(\mathbf{v}_{N}, \vartheta_{N}\right)\right\rangle, \quad{ }^{\forall}\left(\mathbf{v}_{N}, \vartheta_{N}\right) \in X_{N}
$$


Due to (10), this can be written as

$$
\left\langle\mathscr{L}_{0}\left(\mathbf{u}_{h}, \theta_{h}\right),\left(\mathbf{v}_{N}, \vartheta_{N}\right)\right\rangle=\left\langle F_{0}(\overline{\mathbf{u}}, \bar{\theta}),\left(\mathbf{v}_{N}, \vartheta_{N}\right)\right\rangle, \quad{ }^{\forall}\left(\mathbf{v}_{N}, \vartheta_{N}\right) \in X_{N}
$$

The left hand side of (21) can be written as:

$$
\begin{aligned}
\left\langle\mathscr{L}_{0}\left(\mathbf{u}_{h}, \theta_{h}\right),\left(\mathbf{v}_{N}, \vartheta_{N}\right)\right\rangle & =\left\langle\mathscr{S}^{-1}\left(\mathbf{u}_{h}, \theta_{h}\right)-F^{\prime}\left(\mathbf{u}_{N}, \theta_{N}\right)\left(\mathbf{u}_{h}, \theta_{h}\right),\left(\mathbf{v}_{N}, \vartheta_{N}\right)\right\rangle \\
& =\left\langle\nabla\left(\mathbf{u}_{h}, \theta_{h}\right), \nabla\left(\mathbf{v}_{N}, \vartheta_{N}\right)\right\rangle-\left\langle F^{\prime}\left(\mathbf{u}_{N}, \theta_{N}\right)\left(\mathbf{u}_{h}, \theta_{h}\right),\left(\mathbf{v}_{N}, \vartheta_{N}\right)\right\rangle .
\end{aligned}
$$

This gives us the interval version of the Jacobian matrix in Newton's method with respect to the base functions $\Phi^{\alpha}, \Psi^{\alpha}$, and $\phi_{3}^{\alpha}$. The right hand side of (21) forms an interval vector whose elements can be enclosed with upper and lower bounds. Thus the operator $\mathscr{L}_{N}$ is regular when the solution $\left(\mathbf{u}_{h}, \theta_{h}\right) \in X_{N}$ for (21) exists under the guaranteed computation with interval arithmetic.

In order to compute $m_{i}^{(k+1)}$ in (19), we need to estimate $\|\overline{\mathbf{f}}(\mathbf{u}, \theta)\|_{0}$ and $\|\bar{g}(\mathbf{u}, \theta)\|_{0}$ for any $(\mathbf{u}, \theta) \in U$. Pick up an element $(\mathbf{u}, \theta) \equiv\left(\mathbf{u}_{h}+\mathbf{u}_{*}, \theta_{h}+\theta_{*}\right) \in U$ with $\left(\mathbf{u}_{h}, \theta_{h}\right) \in U_{N},\left(\mathbf{u}_{*}, \theta_{*}\right) \in U_{*}$, then we have

$$
\begin{aligned}
\overline{\mathbf{f}}(\mathbf{u}, \theta) & =\mathbf{f}\left(\mathbf{u}_{N}+\mathbf{u}_{h}+\mathbf{u}_{*}, \theta_{N}+\theta_{h}+\theta_{*}\right)+\Delta \mathbf{u}_{N}-\nabla p_{N} \\
& =\mathbf{f}\left(\mathbf{u}_{N}+\mathbf{u}_{h}, \theta_{N}+\theta_{h}\right)+\Delta \mathbf{u}_{N}-\nabla p_{N} \\
& -\frac{1}{\mathscr{P}}\left[\left(\left(\mathbf{u}_{N}+\mathbf{u}_{h}\right) \cdot \nabla\right) \mathbf{u}_{*}+\left(\mathbf{u}_{*} \cdot \nabla\right)\left(\mathbf{u}_{N}+\mathbf{u}_{h}\right)+\left(\mathbf{u}_{*} \cdot \nabla\right) \mathbf{u}_{*}\right]+\mathscr{R} \theta_{*} \mathbf{e}_{z}, \\
\bar{g}(\mathbf{u}, \theta) & =g\left(\mathbf{u}_{N}+\mathbf{u}_{h}+\mathbf{u}_{*}, \theta_{N}+\theta_{h}+\theta_{*}\right)+\Delta \theta_{N} \\
& =g\left(\mathbf{u}_{N}+\mathbf{u}_{h}, \theta_{N}+\theta_{h}\right)+\Delta \theta_{N} \\
& -\left[\left(\left(\mathbf{u}_{N}+\mathbf{u}_{h}\right) \cdot \nabla\right) \theta_{*}+\left(\mathbf{u}_{*} \cdot \nabla\right)\left(\theta_{N}+\theta_{h}\right)+\left(\mathbf{u}_{*} \cdot \nabla\right) \theta_{*}\right]+w_{*} .
\end{aligned}
$$

These forms enable us to estimate the desired norms as follows:

$$
\begin{aligned}
\|\overline{\mathbf{f}}(\mathbf{u}, \theta)\|_{0} & \leq\left\|\mathbf{f}\left(\mathbf{u}_{N}+\mathbf{u}_{h}, \theta_{N}+\theta_{h}\right)+\Delta \mathbf{u}_{N}-\nabla p_{N}\right\|_{0} \\
& +\frac{1}{\mathscr{P}}\left[\left\|\mathbf{u}_{N}+\mathbf{u}_{h}\right\|_{\infty}\left\|\nabla \mathbf{u}_{*}\right\|_{0}+\left\|\mathbf{u}_{*}\right\|_{0}\left\|\nabla\left(\mathbf{u}_{N}+\mathbf{u}_{h}\right)\right\|_{\infty}+\left\|\mathbf{u}_{*}\right\|_{\infty}\left\|\nabla \mathbf{u}_{*}\right\|_{0}\right]+\mathscr{R}\left\|_{*}\right\|_{0} \\
& \leq\left\|\mathbf{f}\left(\mathbf{u}_{N}+\mathbf{u}_{h}, \theta_{N}+\theta_{h}\right)+\Delta \mathbf{u}_{N}-\nabla p_{N}\right\|_{0} \\
& +\frac{1}{\mathscr{P}}\left[\frac{C_{0}}{N+1}\left\|\mathbf{u}_{N}+\mathbf{u}_{h}\right\|_{\infty}+\frac{C_{0}^{2}}{(N+1)^{2}}\left\|\nabla\left(\mathbf{u}_{N}+\mathbf{u}_{h}\right)\right\|_{\infty}\right] m_{1}+\frac{2 C_{0} C_{1}}{(N+1) \sqrt{N}} m_{1}^{2}+\mathscr{R} \frac{C_{0}^{2}}{(N+1)^{2}} m_{2}, \\
\|\bar{g}(\mathbf{u}, \theta)\|_{0} & \leq\left\|g\left(\mathbf{u}_{N}+\mathbf{u}_{h}, \theta_{N}+\theta_{h}\right)+\Delta \theta_{N}\right\|_{0} \\
& +\left[\left\|\mathbf{u}_{N}+\mathbf{u}_{h}\right\|_{\infty}\left\|\nabla \theta_{*}\right\|_{0}+\left\|\mathbf{u}_{*}\right\|_{0}\left\|\nabla\left(\theta_{N}+\theta_{h}\right)\right\|_{\infty}+\left\|\mathbf{u}_{*}\right\|_{\infty}\left\|\nabla \theta_{*}\right\|_{0}\right]+\left\|w_{*}\right\|_{0} \\
& \leq\left\|g\left(\mathbf{u}_{N}+\mathbf{u}_{h}, \theta_{N}+\theta_{h}\right)+\Delta \theta_{N}\right\|_{0} \\
& +\frac{C_{0}}{N+1}\left\|\mathbf{u}_{N}+\mathbf{u}_{h}\right\|_{\infty} m_{2}+\frac{C_{0}^{2}}{(N+1)^{2}}\left\|\nabla\left(\theta_{N}+\theta_{h}\right)\right\|_{\infty} m_{1}+\frac{2 C_{0} C_{1}}{(N+1) \sqrt{N}} m_{1} m_{2}+\frac{C_{0}^{2}}{(N+1)^{2}} m_{1} .
\end{aligned}
$$

Note that upper bounds of $L^{2}$ and $L^{\infty}$ norms for $\left(\mathbf{u}_{N}+\mathbf{u}_{h}, \theta_{N}+\theta_{h}\right) \in U_{N} \subset X_{N}$ can be computed by interval arithmetic, and these calculation may have additional inflations due to crude estimates. Thus we estimate $\left\|\left(I-P_{N}\right) \Pi_{1} \mathscr{S} \bar{F}(\overline{\mathbf{u}}, \bar{\theta})\right\|_{0},\left\|\nabla\left(I-P_{N}\right) \Pi_{1} \mathscr{S} \bar{F}(\overline{\mathbf{u}}, \bar{\theta})\right\|_{0},\left\|\left(I-P_{N}\right) \Pi_{1} \mathscr{S} \bar{F}(\overline{\mathbf{u}}, \bar{\theta})\right\|_{\infty}\left\|\left(I-Q_{N}\right) \Pi_{2} \mathscr{S} \bar{F}(\overline{\mathbf{u}}, \bar{\theta})\right\|_{0}$, and $\left\|\nabla\left(I-Q_{N}\right) \Pi_{2} \mathscr{S} \bar{F}(\overline{\mathbf{u}}, \bar{\theta})\right\|_{0}$ after decomposition of $\Pi_{1} \mathscr{S} \bar{F}(\overline{\mathbf{u}}, \bar{\theta})$ and $\Pi_{2} \mathscr{S} \bar{F}(\overline{\mathbf{u}}, \bar{\theta})$ into finite and infinite parts, which gives us more accurate values of them and efficient estimates in real computations.

\section{Numerical results}

For the interval arithmetic, we use the PROFIL package [2] on Linux Intel Pentium 4 (3.8 GHz) machine.

We set $a^{2}=\frac{1}{8}, b^{2}=\frac{3}{8}$ and $\mathscr{P}=10$ in the numerical experiments with $1 \%$ inflation factor. Then the critical Rayleigh number $\mathscr{R}_{c}=6.75$ can be attained at some special mode $\alpha$ (see [5] for detail). We show several approximation results in figures 1-3. In these figures, the isothermal lines are drawn after adding the conduction solution (2) on the left, and contour lines of speed with streamlines are shown on the right. Note that streamlines for each type never change their shape during the change of relative Rayleigh numbers in short range. We present figures at the same relative Rayleigh number $\mathscr{R} / \mathscr{R}_{c}=1.1$ for easy comparison. 

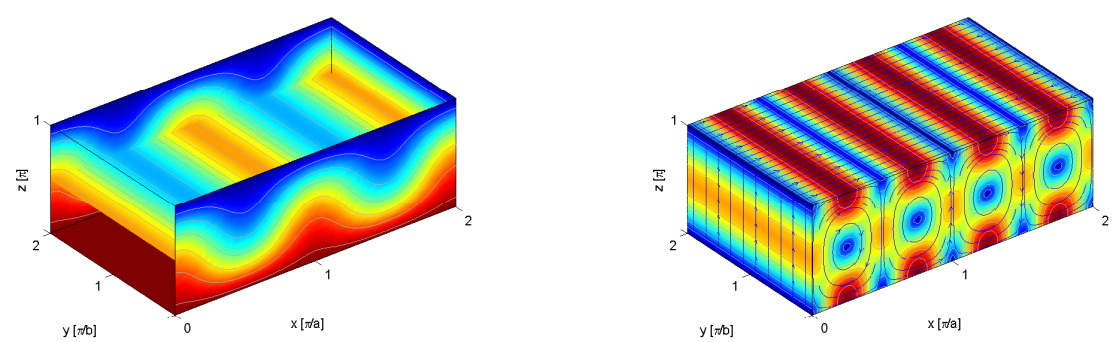

Fig. 1 Isothermal lines, and contour lines of speed with streamlines for roll type at $\mathscr{R} / \mathscr{R}_{c}=1.1$.
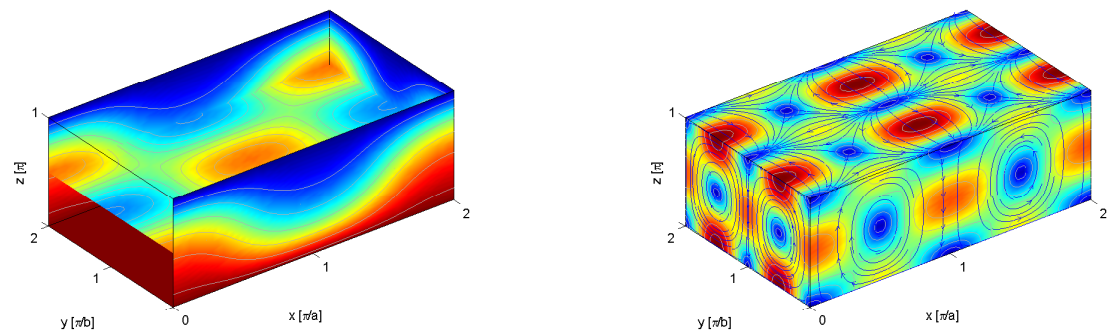

Fig. 2 Isothermal lines, and contour lines of speed with streamlines for rectangular type at $\mathscr{R} / \mathscr{R}_{c}=1.1$.
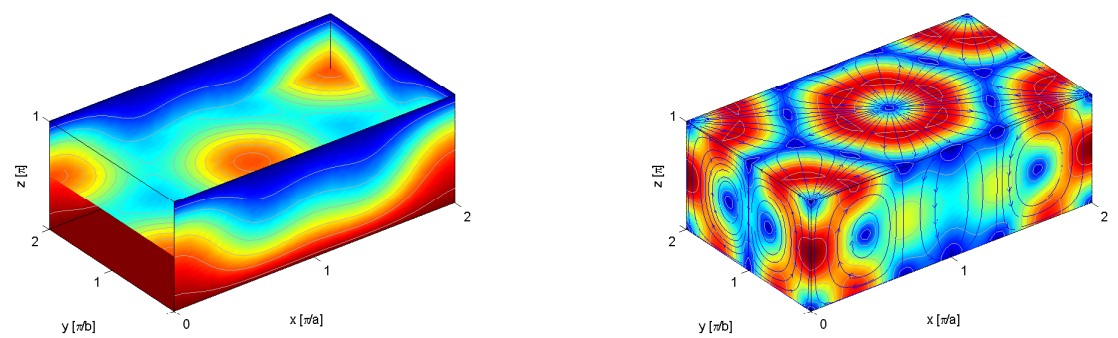

Fig. 3 Isothermal lines, and contour lines of speed with streamlines for hexagonal type at $\mathscr{R} / \mathscr{R}_{c}=1.1$.

In Table 1, Table 2 and Table 3, we illustrate the verification results for each type of solutions with several relative Rayleigh numbers. In these tables, we show the relative Rayleigh number $\mathscr{R} / \mathscr{R}_{c}$, the truncation number $N$, the converged step $k, L^{\infty}$ norms of approximate solutions $\left(\mathbf{u}_{N}, \theta_{N}\right), L^{\infty}$ norms of finite parts $\left(\mathbf{u}_{h}, \theta_{h}\right)$, and the bounds $m_{1}, m_{2}$ of infinite parts. The converged step means the inflated candidate set at step $k-\frac{1}{2}$ includes the new one at step $k$, namely, the verification was completed at the concerning iteration steps. In the roll type case (Table 1), the problem size becomes much smaller due to the elimination of one space variable which comes from the fact that the solutions are independent of that variable. For other types (Table 2, Table 3 ), we can find out the basic symmetry of solutions which make it possible to reduce the size of unknown coefficients.

From these tables, we can make a bifurcation diagram Fig. 4 with respect to the relative Rayleigh number $\mathscr{R} / \mathscr{R}_{c}$ and sum $\left\|\nabla \mathbf{u}_{N}\right\|_{\infty}+\left\|\nabla \theta_{N}\right\|_{\infty}$ of approximate solutions' $L^{\infty}$ norms.

\section{Conclusion}

We could verify several kinds of bifurcating solutions. This should be the first result on the fact that there actually exist exact solutions around approximate solutions drawn in the figures corresponding to the interesting 


\begin{tabular}{|l|r|r|rc|c|c|c|c|}
\hline $\mathscr{R} / \mathscr{R}_{c}$ & $N$ & $k$ & $\left\|\nabla \mathbf{u}_{N}\right\|_{\infty}$ & $\left\|\nabla \theta_{N}\right\|_{\infty}$ & $\left\|\nabla \mathbf{u}_{h}\right\|_{\infty}$ & $\left\|\nabla \theta_{h}\right\|_{\infty}$ & $m_{1}$ & $m_{2}$ \\
\hline \hline 1.01 & 16 & 12 & 0.74 & 0.31 & $5 \times 10^{-11}$ & $2 \times 10^{-10}$ & $1.44 \times 10^{-10}$ & $1.92 \times 10^{-10}$ \\
1.05 & 18 & 29 & 1.66 & 0.72 & $2 \times 10^{-8}$ & $2 \times 10^{-8}$ & $4.06 \times 10^{-9}$ & $3.57 \times 10^{-9}$ \\
1.5 & 44 & 19 & 5.58 & 2.47 & $3 \times 10^{-11}$ & $3 \times 10^{-10}$ & $1.72 \times 10^{-11}$ & $3.33 \times 10^{-11}$ \\
\hline
\end{tabular}

Table 1 Verification results for roll type solutions.

\begin{tabular}{|c|c|c|c|c|c|c|c|c|}
\hline $\mathscr{R} / \mathscr{R}_{c}$ & $N$ & $k$ & $\left\|\nabla \mathbf{u}_{N}\right\|_{\infty}$ & $\left\|\nabla \theta_{N}\right\|_{\infty}$ & $\mid \nabla \mathbf{u}_{h} \|_{\infty}$ & $\mid \nabla \theta_{h} \|_{\infty}$ & $m_{1}$ & $m_{2}$ \\
\hline 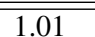 & $\overline{\overline{16}}$ & 7 & 0.93 & 0.38 & $2 \times 10^{-11}$ & $3 \times 10^{-11}$ & $2.37 \times 10^{-12}$ & $1.01 \times 10^{-11}$ \\
\hline 1.05 & 16 & 10 & 2.20 & 0.96 & $2 \times 10^{-8}$ & $2 \times 10^{-7}$ & $2.91 \times 10^{-9}$ & $1.09 \times 10^{-8}$ \\
\hline $\begin{array}{l}1.00 \\
1.1\end{array}$ & 24 & 11 & 3.26 & 1.47 & $4 \times 10^{-10}$ & $3 \times 10^{-9}$ & $8.33 \times 10^{-13}$ & $5.84 \times 10^{-12}$ \\
\hline 1.2 & 28 & 33 & 4.99 & 2.27 & $2 \times 10^{-10}$ & $2 \times 10^{-9}$ & $4.60 \times 10^{-12}$ & $3.96 \times 10^{-11}$ \\
\hline
\end{tabular}

Table 2 Verification results for rectangular type solutions.

\begin{tabular}{|l|r|r|rc|c|c|cc|}
\hline $\mathscr{R} / \mathscr{R}_{C}$ & $N$ & $k$ & $\left\|\nabla \mathbf{u}_{N}\right\|_{\infty}$ & $\left\|\nabla \theta_{N}\right\|_{\infty}$ & $\left\|\nabla \mathbf{u}_{h}\right\|_{\infty}$ & $\left\|\nabla \theta_{h}\right\|_{\infty}$ & $m_{1}$ & $m_{2}$ \\
\hline \hline 1.01 & 16 & 7 & 1.12 & 0.47 & $2 \times 10^{-10}$ & $6 \times 10^{-10}$ & $4.53 \times 10^{-11}$ & $1.93 \times 10^{-10}$ \\
1.05 & 16 & 15 & 2.70 & 1.21 & $2 \times 10^{-6}$ & $2 \times 10^{-6}$ & $3.22 \times 10^{-8}$ & $1.08 \times 10^{-7}$ \\
1.1 & 24 & 19 & 4.09 & 1.88 & $4 \times 10^{-9}$ & $3 \times 10^{-8}$ & $2.50 \times 10^{-11}$ & $1.68 \times 10^{-10}$ \\
\hline
\end{tabular}

Table 3 Verification results for hexagonal type solutions.

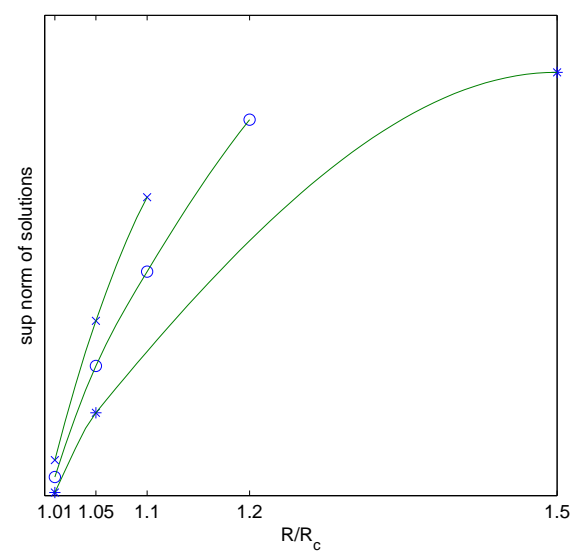

Fig. 4 Bifurcation diagram. $\times$ for hexagonal, $\circ$ for rectangular, and $*$ for roll type cases.

natural phenomena. Due to the limit of our computational power up to now, we proved only small part (Fig. 4) of bifurcation diagram except for the roll type solutions. This could be enhanced using parallel computation after update of PROFIL package into parallel version (not available now) or increasing physical memories for computation on some large scale computers.

More interesting problem would be the verification of the bifurcation point such that suggested in [5], which is more complicated problem than the usual. We believe that these interesting and important problems could be resolved in the near future.

\section{References}

1. Fabrice Caini and Philippe Gelene, Rayleigh-benard convection, http://www.enseeiht.fr/hmf/travaux/CD9598/ travaux/optmfn/BES_PHOENICS/96-97/Caini-Gelene/RB_convectio_tur.html; accessed Dec 2, 2006.

2. Siegfried M. Rump (Head), Profil, http://www.ti3.tu-harburg.de/Software/PROFILEnglisch.html; accessed Dec 4 , 2006, Institute for Reliable Computing.

3. Paul Manneville, Rayleigh-Bénard convection, thirty years of experimental, theoretical, and modeling work, http://www . ladhyx.polytechnique.fr/people/pops/benard.pdf; accessed Feb 16, 2005. 
4. Mitsuhiro T. Nakao, Yoshitaka Watanabe, Nobito Yamamoto, and Takaaki Nishida, Some computer assisted proofs for solutions of the heat convection problems, Reliable Computing 9 (2003), no. 5, 359-372.

5. Takaaki Nishida, Tsutomu Ikeda, and Hideaki Yoshihara, Mathematical modeling and numerical simulation in continuum mechanics, Lecture Notes in Computational Sciences and Engineering, vol. 19, ch. Pattern Formation of Heat Convection Problems, pp. 209-218, Springer, 2002.

6. Michael Plum, Explicit $\mathrm{H}_{2}$-estimates and pointwise bounds for solutions of second-order elliptic boundary value problems, Journal of Mathematical Analysis and Applications 165 (1992), 36-61.

7. Yoshitaka Watanabe, Nobito Yamamoto, Mistuhiro T. Nakao, and Takaaki Nishida, A numerical verification of nontrivial solutions for the heat convection problem, Journal of Mathematical Fluid Mechanics 6 (2004), 1-20. 


\section{List of MHF Preprint Series, Kyushu University 21st Century COE Program \\ Development of Dynamic Mathematics with High Functionality}

\section{MHF2005-1 Hideki KOSAKI}

Matrix trace inequalities related to uncertainty principle

MHF2005-2 Masahisa TABATA

Discrepancy between theory and real computation on the stability of some finite element schemes

MHF2005-3 Yuko ARAKI \& Sadanori KONISHI

Functional regression modeling via regularized basis expansions and model selection

MHF2005-4 Yuko ARAKI \& Sadanori KONISHI

Functional discriminant analysis via regularized basis expansions

MHF2005-5 Kenji KAJIWARA, Tetsu MASUDA, Masatoshi NOUMI, Yasuhiro OHTA \& Yasuhiko YAMADA

Point configurations, Cremona transformations and the elliptic difference Painlevé equations

MHF2005-6 Kenji KAJIWARA, Tetsu MASUDA, Masatoshi NOUMI, Yasuhiro OHTA \& Yasuhiko YAMADA

Construction of hypergeometric solutions to the $q$ - Painlevé equations

MHF2005-7 Hiroki MASUDA

Simple estimators for non-linear Markovian trend from sampled data:

I. ergodic cases

MHF2005-8 Hiroki MASUDA \& Nakahiro YOSHIDA

Edgeworth expansion for a class of Ornstein-Uhlenbeck-based models

MHF2005-9 Masayuki UCHIDA

Approximate martingale estimating functions under small perturbations of dynamical systems

MHF2005-10 Ryo MATSUZAKI \& Masayuki UCHIDA

One-step estimators for diffusion processes with small dispersion parameters from discrete observations

MHF2005-11 Junichi MATSUKUBO, Ryo MATSUZAKI \& Masayuki UCHIDA

Estimation for a discretely observed small diffusion process with a linear drift

MHF2005-12 Masayuki UCHIDA \& Nakahiro YOSHIDA

AIC for ergodic diffusion processes from discrete observations 
MHF2005-13 Hiromichi GOTO \& Kenji KAJIWARA

Generating function related to the Okamoto polynomials for the Painlevé IV equation

MHF2005-14 Masato KIMURA \& Shin-ichi NAGATA

Precise asymptotic behaviour of the first eigenvalue of Sturm-Liouville problems with large drift

MHF2005-15 Daisuke TAGAMI \& Masahisa TABATA

Numerical computations of a melting glass convection in the furnace

MHF2005-16 Raimundas VIDŪNAS

Normalized Leonard pairs and Askey-Wilson relations

MHF2005-17 Raimundas VIDŪNAS

Askey-Wilson relations and Leonard pairs

MHF2005-18 Kenji KAJIWARA \& Atsushi MUKAIHIRA

Soliton solutions for the non-autonomous discrete-time Toda lattice equation

MHF2005-19 Yuu HARIYA

Construction of Gibbs measures for 1-dimensional continuum fields

MHF2005-20 Yuu HARIYA

Integration by parts formulae for the Wiener measure restricted to subsets in $\mathbb{R}^{d}$

MHF2005-21 Yuu HARIYA

A time-change approach to Kotani's extension of Yor's formula

MHF2005-22 Tadahisa FUNAKI, Yuu HARIYA \& Mark YOR

Wiener integrals for centered powers of Bessel processes, I

MHF2005-23 Masahisa TABATA \& Satoshi KAIZU

Finite element schemes for two-fluids flow problems

MHF2005-24 Ken-ichi MARUNO \& Yasuhiro OHTA

Determinant form of dark soliton solutions of the discrete nonlinear Schrödinger equation

MHF2005-25 Alexander V. KITAEV \& Raimundas VIDŪNAS

Quadratic transformations of the sixth Painlevé equation

MHF2005-26 Toru FUJII \& Sadanori KONISHI

Nonlinear regression modeling via regularized wavelets and smoothing parameter selection

MHF2005-27 Shuichi INOKUCHI, Kazumasa HONDA, Hyen Yeal LEE, Tatsuro SATO, Yoshihiro MIZOGUCHI \& Yasuo KAWAHARA

On reversible cellular automata with finite cell array 
MHF2005-28 Toru KOMATSU

Cyclic cubic field with explicit Artin symbols

MHF2005-29 Mitsuhiro T. NAKAO, Kouji HASHIMOTO \& Kaori NAGATOU

A computational approach to constructive a priori and a posteriori error estimates for finite element approximations of bi-harmonic problems

MHF2005-30 Kaori NAGATOU, Kouji HASHIMOTO \& Mitsuhiro T. NAKAO Numerical verification of stationary solutions for Navier-Stokes problems

MHF2005-31 Hidefumi KAWASAKI

A duality theorem for a three-phase partition problem

MHF2005-32 Hidefumi KAWASAKI

A duality theorem based on triangles separating three convex sets

MHF2005-33 Takeaki FUCHIKAMI \& Hidefumi KAWASAKI

An explicit formula of the Shapley value for a cooperative game induced from the conjugate point

MHF2005-34 Hideki MURAKAWA

A regularization of a reaction-diffusion system approximation to the two-phase Stefan problem

MHF2006-1 Masahisa TABATA

Numerical simulation of Rayleigh-Taylor problems by an energy-stable finite element scheme

MHF2006-2 Ken-ichi MARUNO \& G R W QUISPEL

Construction of integrals of higher-order mappings

MHF2006-3 Setsuo TANIGUCHI

On the Jacobi field approach to stochastic oscillatory integrals with quadratic phase function

MHF2006-4 Kouji HASHIMOTO, Kaori NAGATOU \& Mitsuhiro T. NAKAO

A computational approach to constructive a priori error estimate for finite element approximations of bi-harmonic problems in nonconvex polygonal domains

MHF2006-5 Hidefumi KAWASAKI

A duality theory based on triangular cylinders separating three convex sets in $R^{n}$

MHF2006-6 Raimundas VIDŪNAS

Uniform convergence of hypergeometric series

MHF2006-7 Yuji KODAMA \& Ken-ichi MARUNO

N-Soliton solutions to the DKP equation and Weyl group actions 
MHF2006-8 Toru KOMATSU

Potentially generic polynomial

MHF2006-9 Toru KOMATSU

Generic sextic polynomial related to the subfield problem of a cubic polynomial

MHF2006-10 Shu TEZUKA \& Anargyros PAPAGEORGIOU

Exact cubature for a class of functions of maximum effective dimension

MHF2006-11 Shu TEZUKA

On high-discrepancy sequences

MHF2006-12 Raimundas VIDŪNAS

Detecting persistent regimes in the North Atlantic Oscillation time series

MHF2006-13 Toru KOMATSU

Tamely Eisenstein field with prime power discriminant

MHF2006-14 Nalini JOSHI, Kenji KAJIWARA \& Marta MAZZOCCO

Generating function associated with the Hankel determinant formula for the solutions of the Painlevé IV equation

MHF2006-15 Raimundas VIDŪNAS

Darboux evaluations of algebraic Gauss hypergeometric functions

MHF2006-16 Masato KIMURA \& Isao WAKANO

New mathematical approach to the energy release rate in crack extension

MHF2006-17 Toru KOMATSU

Arithmetic of the splitting field of Alexander polynomial

MHF2006-18 Hiroki MASUDA

Likelihood estimation of stable Lévy processes from discrete data

MHF2006-19 Hiroshi KAWABI \& Michael RÖCKNER

Essential self-adjointness of Dirichlet operators on a path space with Gibbs measures via an SPDE approach

MHF2006-20 Masahisa TABATA

Energy stable finite element schemes and their applications to two-fluid flow problems

MHF2006-21 Yuzuru INAHAMA \& Hiroshi KAWABI

Asymptotic expansions for the Laplace approximations for Itô functionals of Brownian rough paths

MHF2006-22 Yoshiyuki KAGEI

Resolvent estimates for the linearized compressible Navier-Stokes equation in an infinite layer 
MHF2006-23 Yoshiyuki KAGEI

Asymptotic behavior of the semigroup associated with the linearized

compressible Navier-Stokes equation in an infinite layer

MHF2006-24 Akihiro MIKODA, Shuichi INOKUCHI, Yoshihiro MIZOGUCHI \& Mitsuhiko FUJIO

The number of orbits of box-ball systems

MHF2006-25 Toru FUJII \& Sadanori KONISHI

Multi-class logistic discrimination via wavelet-based functionalization and model selection criteria

MHF2006-26 Taro HAMAMOTO, Kenji KAJIWARA \& Nicholas S. WITTE

Hypergeometric solutions to the $q$-Painlevé equation of type $\left(A_{1}+A_{1}^{\prime}\right)^{(1)}$

MHF2006-27 Hiroshi KAWABI \& Tomohiro MIYOKAWA

The Littlewood-Paley-Stein inequality for diffusion processes on general metric spaces

MHF2006-28 Hiroki MASUDA

Notes on estimating inverse-Gaussian and gamma subordinators under highfrequency sampling

MHF2006-29 Setsuo TANIGUCHI

The heat semigroup and kernel associated with certain non-commutative

harmonic oscillators

MHF2006-30 Setsuo TANIGUCHI

Stochastic analysis and the KdV equation

MHF2006-31 Masato KIMURA, Hideki KOMURA, Masayasu MIMURA, Hidenori MIYOSHI, Takeshi TAKAISHI \& Daishin UEYAMA

Quantitative study of adaptive mesh FEM with localization index of pattern

MHF2007-1 Taro HAMAMOTO \& Kenji KAJIWARA

Hypergeometric solutions to the $q$-Painlevé equation of type $A_{4}^{(1)}$

MHF2007-2 Kouji HASHIMOTO, Kenta KOBAYASHI \& Mitsuhiro T. NAKAO

Verified numerical computation of solutions for the stationary Navier-Stokes equation in nonconvex polygonal domains

MHF2007-3 Kenji KAJIWARA, Marta MAZZOCCO \& Yasuhiro OHTA A remark on the Hankel determinant formula for solutions of the Toda equation

MHF2007-4 Jun-ichi SATO \& Hidefumi KAWASAKI

Discrete fixed point theorems and their application to Nash equilibrium

MHF2007-5 Mitsuhiro T. NAKAO \& Kouji HASHIMOTO

Constructive error estimates of finite element approximations for non-coercive elliptic problems and its applications 
MHF2007-6 Kouji HASHIMOTO

A preconditioned method for saddle point problems

MHF2007-7 Christopher MALON, Seiichi UCHIDA \& Masakazu SUZUKI

Mathematical symbol recognition with support vector machines

MHF2007-8 Kenta KOBAYASHI

On the global uniqueness of Stokes' wave of extreme form

MHF2007-9 Kenta KOBAYASHI

A constructive a priori error estimation for finite element discretizations in a non-convex domain using singular functions

MHF2007-10 Myoungnyoun KIM, Mitsuhiro T. NAKAO, Yoshitaka WATANABE \& Takaaki NISHIDA

A numerical verification method of bifurcating solutions for 3-dimensional Rayleigh-Bénard problems 